\title{
Research of Image Enhancement Algorithm under Inclement Weather
}

\author{
Zhenyu Liu ${ }^{1, a}$, Hairong Jiang ${ }^{1, b}$ and Hewen $\mathrm{Xu}^{2, \mathrm{c}}$ \\ ${ }^{1}$ School of Information Science and Engineering, Shenyang University of Technology, Shenyang \\ 110870, China; \\ ${ }^{2}$ Baicheng Weapon Test Center ,Baicheng 137000, China. \\ aliu_zhenyu0419@sina.com, b742667749@qq.com, '693263641@qq.com
}

\begin{abstract}
Keywords: Adaptive white balance; image dehazing; dust image enhancement
\end{abstract}
\begin{abstract}
To make the image details in smoggy and dusty weather clear without reducing the quality of clear parts, is the key point of outdoor static image restoration. This paper put forward an image enhancement algorithm that can handle haze and dust image at once. First, use self-adaptive white balance algorithm to correct the color cast. Second, put forward a bright region segmentation method to revise transmittance which results in distortion in bright area based on dark channel prior. Third, use adaptive gamma technology to further correct the transmittance. Experiments show this method can recover more degraded image information than existing estimation methods.
\end{abstract}

\section{Introduction}

Images under inclement weather like haze and dust often perform severe degradation due to suspended particles' absorption and scattering processes. It not only attenuates reflected light from the outdoor scenes, but also penetrates the atmosphere light. These degraded images directly reduce the performance of object recognition[1], obstacle detection [2,3], intelligence transportation [4,5] and other systems.

In order to improve the sharpness of degraded images, numerous effective algorithms have been proposed. Tan [6] wiped off fog by maximizing local contrast. But this algorithm can easily cause image supersaturated and distortion. He et al.[7] put forward a single image haze removal method based on dark channel prior. But it doesn't fit for images that are not bright enough. Yan Ting et al.[8] used global histogram algorithm and POSHE ( partially overlapping sub-block histogram equalization algorithm) to enhance image. This method effectively improves dust image contrast, but easy to bring color distortion and is ineffective on distant image.

This paper raises a new algorithm that can increase the sharpness of hazy image and dusty image at once. First, use adaptive white balance to deal with color distortion and reduce the impact of dust and haze upon image color. Second, bring forward a bright region segmentation method to revise transmission which results in distortion in bright area based on dark channel prior. Then use adaptive gamma technology to further correct the transmittance to get clear and natural image.

\section{The Image Dehazing Algorithm}

\subsection{Atmospheric Scattering Model}

Currently, most haze image enhancement algorithms are based on atmospheric scattering model.

$$
I(x)=J(x) t(x)+A(1-t(x))
$$

In the formula, $I(x)$ is the degraded image. $t(x)(0 \leq t(x) \leq 1)$ represents the transmission that describes lights reaching the camera without scattering. $J(x)$ means the scene radiance that is the recovered clear image. A is the global atmospheric light. The aim of dehazing is to restore $J(x), t(x)$ and A from $I(x)$.

\subsection{The Dark Channel Prior Algorithm}

He et al found the dark channel prior by observing numerous statistical features of haze-free images. He found in a non-sky haze-free image, one of those pixels' color channels in each block will nearly reach 0 if the image is divided into many sub blocks. 


$$
J^{\operatorname{dark}}(x)=\min _{y \in \Omega(x)}\left(\min _{c \in\{R, G, B\}} J^{c}(y)\right)=0
$$

$J^{c}$ is one color channel of $\mathrm{J}$. c is one of the R,G,B channels. $\Omega(x)$ is a local patch centered at X. $J^{\text {dark }}$ is the dark channel of J. For $A^{c}$ is always positive, the transmission can be derived from equation (2):

$$
t(x)=1-w \min _{y \in \Omega(x)}\left(\min _{c \in\{R, G, B\}}\left(\frac{I^{c}(y)}{A^{c}}\right)\right)
$$

The picture would be seen unnatural and lose the depth of field if completely remove the haze. Then add a constant $w=0.95$ to retain part of haze that covers distant scene. Finally, the scene radiance $\mathrm{J}$ is:

$$
J(x)=\frac{I(x)-A}{\max \left(t(x), t_{0}\right)}+A
$$

It is important to note that the recovered scene radiance can be prone to noise production when $\mathrm{t}(\mathrm{x})$ is close to 0 . This could make the image include noise. Thus, an inferior limit is set as $t_{0}=0.1$.

\section{Proposed Algorithm}

Studies show that hazy image always drift to gray, dust image red, backlight black. A new algorithm is proposed to dispose both haze and dust images. First, use adaptive white balance to deal with color distortion. Then enhance image color based on improved dark channel prior. Finally, get clear and natural restored image.

\subsection{Adaptive White Balance}

Adaptive white balance can eliminate color shift by estimating scene luminous via image itself making the image more natural. It usually selects the brightest one in referenced white points as the channel gain. The way to acquire the white point is similar to the atmospheric optical in dark channel prior. This paper will pick the atmospheric light A as the white spot to calibrate image. He[7] pick the highest one in top $0.1 \%$ pixels as A. However, if just choose one point, the value of A in each channel could all close to 255, which will result in undesirable color cast. The atmospheric light refers to the ambient illumination in infinite distance usually choose from the upper part in a image. This paper chooses the mean value of top $0.1 \%$ brightest pixels from the upper $25 \%$ height part as $\mathrm{A}$. If there is almost none sky region in an image, we assume the top of the image is the farthest. After getting A, put $\mathrm{A}$ as the white point. To make the brightness of the final image in keeping with original one, use $\mathrm{A}$ as the reference to calculate the gain of each channel and correct the color temperature.

$$
\left\{\begin{array}{l}
R^{\prime}=\frac{A}{R_{\text {ave }}} \times R \\
G^{\prime}=\frac{A}{G_{\text {ave }}} \times G \\
B^{\prime}=\frac{A}{B_{\text {ave }}} \times B
\end{array}\right.
$$

In formula (5), $R_{\text {ave }} 、 G_{\text {ave }}$ and $B_{\text {ave }}$ are the mean of R、G、B channels of the top $0.1 \%$ brightest pixels in dark channel located in upper 25\% height of the image. $R^{\prime} 、 G^{\prime}$ and $B^{\prime}$ are the value of pixels

in three channels after white balance. $\frac{A}{R_{\text {ave }}} 、 \frac{A}{G_{\text {ave }}} 、 \frac{A}{B_{\text {ave }}}$ are the channel gain of $\mathrm{R} 、 \mathrm{G} 、 \mathrm{~B}$. After acquiring the gain of each channel, adjust all pixels of the original image.

\subsection{The Improved Dark Channel Prior Algorithm}

He's algorithm[7] is based on dark channel assumption. But when the scene approximates the air layer and no shadow covered, it is invalid. Because the sky doesn't meet the dark channel prior theory, its actual transmission is much bigger than estimated. The undersized transmission brings the wrong restored color and leads to a smaller J(x),resulting in shading effect at the edge. First, set a threshold S to judge the bright block, if difference between the atmospheric light and the dark channel lower than $\mathrm{S}$, then judge the block as bright area, otherwise, is not the bright. $\mathrm{S}$ is equal to 45 after experiments verification. For areas of $|A-I(x)| \leq 45$, judge them as bright areas and recalculate the transmission. 
As the bright area doesn't satisfy the dark channel prior:

$$
J^{\operatorname{dark}}(x)=\min _{y \in \Omega(x)}\left(\min _{c \in\{R, G, B\}} J^{c}(y)\right) \neq 0
$$

So the transmission is calculated as follow:

$$
t_{\text {refine }}(x)=\min \left(\beta\left(1-\min _{y \in \Omega(x)}\left(\min _{c \in\{R, G, B\}}\left(\frac{I^{c}(y)}{A^{c}}\right)\right)\right), 1\right)
$$

$\beta$ is the correction coefficient. In order to make the transmission continuous, $\beta$ is equal to $0.35 * \mathrm{~A}$ after abundant tests. In formula(7), 1 is set to prevent the overflow of the corrected transmission. The modified transmission is:

$$
t_{f}(x)=\left\{\begin{array}{l}
t_{\text {refine }}(x),|A-I(x)| \leq 45 \\
t(x), \quad|A-I(x)|>45
\end{array}\right.
$$

The dark channel of the input image is acquired by the minimum value of RGB channel. In general, the lower the dark channel value, the thinner the haze. However, most blue hue of the spectrum in a dust image is absorbed by the atmospheric particles. As shown in figure 1, the histogram of the dark channel is similar to the blue channel. Due to the low intensity of blue channel, the dark channel value of dust image is relatively small. Therefore, the dust concentration processed by dark channel prior often perceive less than reality. Lacking of blue hue leads to an inaccurate dark channel value. To receive the best results, use adaptive gamma correction to adjust the transmission. It redistributes the histogram by adjusting the adaptive parameters in a certain range. The refined transmission is:

$$
t^{\prime}=\left(I_{\max }\right)\left(\frac{t_{f}(x)}{I_{\max }}\right)^{\alpha} \quad, \alpha=\left\{\begin{array}{c}
1+\left(\frac{h}{I_{\max }}\right), \quad h \geq H \\
1, \quad h<H
\end{array}\right.
$$

$I_{\max }$ is the maximum intensity value of input image, $\alpha$ is the adaptive parameter, $\mathrm{h}$ is the intensity value when the cumulative probability density is 0.1 . $\mathrm{H}$ is the threshold of the adaptive parameter set to 120 empirically. Through the adaptive gamma correction operation, we can acquire the clear restored image $J(x)$ by correcting the value of transmission.

$$
J(x)=\frac{I(x)-A}{\max \left(t^{\prime}(x), t_{0}\right)}+A
$$
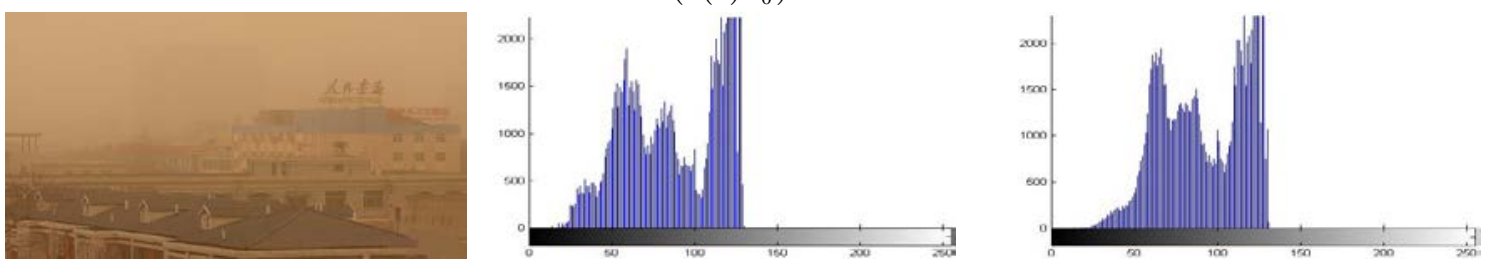

(a)The dust image
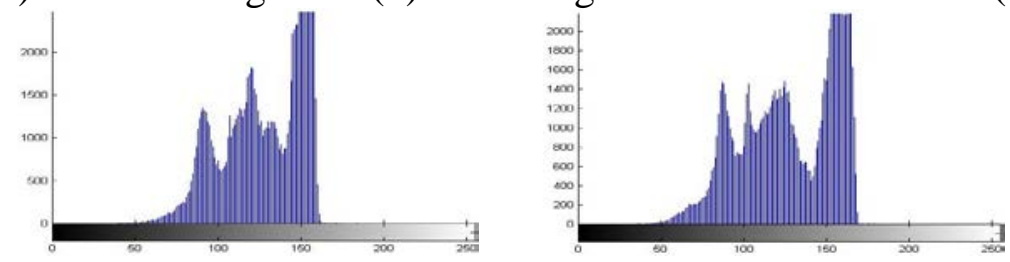

(e)The histogram of the red channel (f) The histogram of the green channel

Fig. 1 Comparison of the image histogram

\section{Experimental Results and Analysis}

All the experimental pictures are from network. Fig.2 and fig.3 are the comparisons of dust and haze image processing effects respectively.As shown in fig.2, compared with Yan ting' $s$ algorithm[8], the restored image in this paper looks natural and clear, while obvious distortion in the sky area can be seen in Yan's method. Fig. 3 is the comparisons results of image dehazing. Compared with He's[7] work, the sky area is better processed, effectively improved the distorted sky region in He's algorithm and better restored majority scene information even the details and the color. As 
shown in fig.2 and fig.3, comparisons of the enhanced results between this paper and the existing methods verify the effectiveness and robustness of the proposed approach.

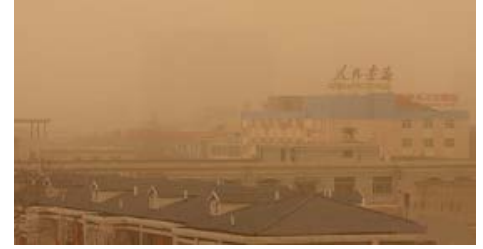

(a) Dust image

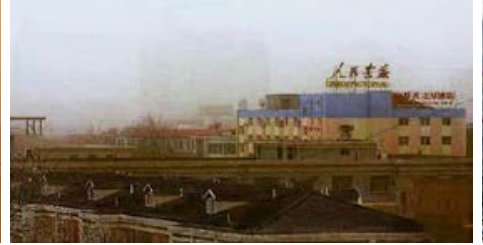

(b) Our resul

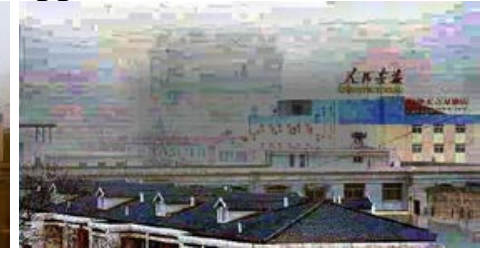

(c) Result of POSHE[8]

Fig.2. Comparison results of dust image

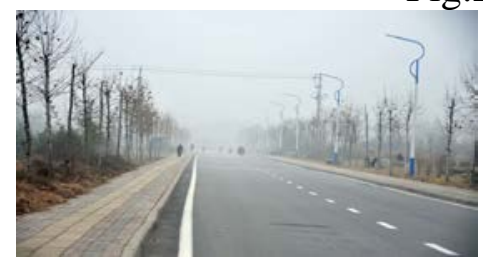

(a) Haze image

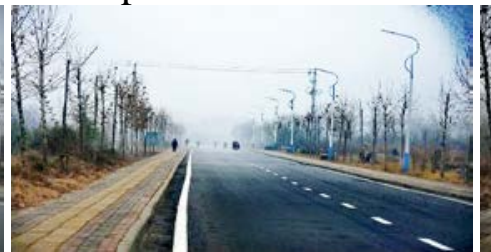

(b) Our result

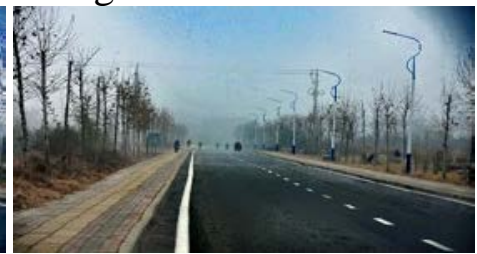

(c) He’s[7]result

Fig.3.Comparison results of image dehazing

\section{Acknowledgments}

This paper is supported by these two programs.Project 1: Industrial and technological projects of Shenyang Science and Technology Bureau, research and development of multi-channel video enhancement system (F14-073-2-00); project 2: Liaoning Provincial Department of education project, research of the workpiece sorting method based on machine vision (L2014041).

Communication author: Hairong Jiang,742667749@qq.com.

\section{References}

[1] R. C. Luo , C. L. Chun. Multisensor fusion-based concurrent environment mapping and moving object detection for intelligent service robotics [J]. IEEE Transactions on Industrial Electronics, 2014,61(8):4043-4051.

[2] H.Rezaee and F. Abdollahi,A decentralized cooperative control scheme with obstacle avoidance for a team of mobile robots [J]. IEEE Transactions on Industrial Electronics, 2014, 61(1): 347 - 354.

[3] Fan Guo, Jin Tang, Zi-Xing Cai. Image dehazing based on haziness analysis[J]. International journal of automation and computing,2014,11(1):78-86.

[4] S. C. Huang, B. H. Chen and Y. J. Cheng. An efficient visibility enhancement algorithm for road scenes captured by intelligent transportation systems [J].IEEE Transactions on Intelligent Transportation Systems, 2014,15(5): 2321 - 2332.

[5] Li Qingwu, Cheng Haisu ,Zhou Yan. Road Vehicle Monitoring System Based on Intelligent Visual Internet of Things [J]. Journal of Sensors, 2015:1-16.

[6] R. Tan, Visibility in bad weather from a single image[C]. IEEE International Conference on Computer Vision and Pattern Recognition, 2008, pp. 1 - 8.

[7] He KM, Sun J, Tang XO. Single image haze removal using dark channel prior[J]. IEEE Transactions on Pattern Analysis and Machine Intelligence,2011,33(12):2341-2353.

[8] Yan Ting et al. Video image enhancement method research in the dust environment [J]. Lasernal, 2014, 35(4):23-25. 